PROCEEDINGS OF THE

AMERICAN MATHEMATICAL SOCIETY

Volume 130, Number 4, Pages 1153-1163

S 0002-9939(01)06295-5

Article electronically published on October 12, 2001

\title{
PROBLÈMES DE PETITES VALEURS PROPRES SUR LES SURFACES DE COURBURE MOYENNE CONSTANTE
}

\author{
PHILIPPE CASTILLON
}

(Communicated by Jozef Dodziuk)

\begin{abstract}
This paper deals with the spectra of the Laplace and stability operators of a constant mean curvature surface in the hyperbolic space. In a preceding work, the author described the essential spectra of these operators, assuming that the surface is of finite total curvature. In this paper, we prove that these two operators have a finite number of eigenvalues below their essential spectra.
\end{abstract}

\section{INTRODUCTION}

Soit $\mathbf{M}^{3}(c)$ un espace forme de dimension 3 et de courbure $c \leq 0$, et $i: M^{2} \hookrightarrow$ $\mathbf{M}^{3}(c)$ une immersion de courbure moyenne constante $h$. La surface $M$ est point critique de la fonctionnelle d'aire pour les déformations qui préservent le volume. On associe à ce problème variationnel l'opérateur de stabilité $S$ de $M$ qui peut être vu comme la hessienne de la fonctionnelle d'aire (cf. par exemple [B-dC-E]). On a $S=\Delta-2\left(h^{2}+c\right)-\left|\mathcal{A}^{0}\right|^{2}$, où $\mathcal{A}^{0}$ est la seconde forme fondamentale à trace nulle. On définit à partir de $S$ l'indice de Morse de $M$ de la façon suivante: pour tout domaine $\Omega$ relativement compact dans $M$ on note $\operatorname{Ind}(\Omega)$ le nombre de valeurs propres négatives de $S$ sur $\Omega$ avec conditions de Dirichlets sur $\partial \Omega$, puis on définit $\operatorname{Ind}(M)=\sup \{\operatorname{Ind}(\Omega) \mid \Omega \subset M\}$.

Dans cet article, on s'intéresse aux relations entre la géométrie de $M$ et les propriétés spectrales du laplacien et de l'opérateur de stabilité. Dans ce cadre, la plupart des études relient l'indice de Morse de $M$ à la courbure totale (cf. [FC, dC-dS, Be-dC-S1, Ca1]). On a en particulier les résultats suivants:

Théorème 0.1 (cf. [FC] et [dC-dS] $)$. Si $h^{2}+c=0$ alors

$$
\int_{M}\left|\mathcal{A}^{0}\right|^{2} d v<\infty \Leftrightarrow \operatorname{Ind}(M)<\infty .
$$

Théorème 0.2 (cf. Be-dC-S1]). Si $h^{2}+c<0$ alors

$$
\int_{M}\left|\mathcal{A}^{0}\right|^{2} d v<\infty \Rightarrow \operatorname{Ind}(M)<\infty
$$

Received by the editors October 11, 2000.

2000 Mathematics Subject Classification. Primary 53C42, 53A10, 58J50; Secondary 58J35.

Key words and phrases. Surfaces de courbure moyenne constante, opérateur de stabilité, théorie spectrale. 
Les exemples de surfaces étudiés par A. da Silveira montrent que la réciproque est fausse (cf. dS $)$.

Lorsque $h^{2}+c=0$ et $\int_{M}\left|\mathcal{A}^{0}\right|^{2} d v<\infty$, la surface $M$ est conforme à une surface de Riemann privée d'un nombre fini de points et sa courbure tend vers 0 à l'infini. La géométrie de $M$ est donc de type euclidien. En particulier, la croissance du volume est quadratique et on $\operatorname{ainf}\left(\sigma_{\text {ess }}(S)\right)=0$. On peut donc voir le théorème 0.1 comme un résultat de finitude sur le nombre d'états bornés d'un opérateur de Schrödinger.

Lorsque $h^{2}+c<0$ et $\int_{M}\left|\mathcal{A}^{0}\right|^{2} d v<\infty$, la surface $M$ est conforme à une surface de Riemann privée d'un nombre fini de disques et sa courbure tend vers $h^{2}+c$ à l'infini. La géométrie de $M$ est donc de type hyperbolique. Comme conséquence, on a que $\inf \left(\sigma_{\text {ess }}(\Delta)\right)=-\frac{h^{2}+c}{4}>0$ et $\inf \left(\sigma_{\text {ess }}(S)\right)=-\frac{9}{4}\left(h^{2}+c\right)>0$ (les spectres essentiels de $\Delta$ et $S$ sont même complètement déterminés; cf. [Ca2]). En dehors du spectre essentiel, les spectres de $\Delta$ et $S$ ne comprennent que des valeurs propres de multiplicités finies isolées dans le spectre; par analogie avec la géométrie hyperbolique, on les appellera les petites valeurs propres de $\Delta$ et $S$.

Du point de vue de la théorie spectrale, le problème naturel qui se pose est celui de la finitude du nombre de petites valeurs propres de $\Delta$ et $S$. L'infimum du spectre essentiel de $S$ étant strictement positif, et $\operatorname{Ind}(M)$ correspondant au nombre de valeurs propres négatives de $S$, le théorème 0.2 ne répond pas à cette question. On montre dans cet article le résultat suivant:

Théorème 0.3. Soit $i: M^{2} \hookrightarrow \mathbf{M}^{3}(c)$ une immersion de courbure moyenne constante $h$ telle que $h^{2}+c<0$ et soit $b=\sqrt{-\left(h^{2}+c\right)}$. Si $\int_{M}\left|\mathcal{A}^{0}\right|^{2} d v<\infty$, alors:

$$
\begin{aligned}
& \text { i. } \sigma_{\text {ess }}(\Delta)=\left[\frac{b^{2}}{4}, \infty\right) \text { et } \mathcal{N}_{\frac{b^{2}}{4}}(\Delta)<\infty, \\
& \text { ii. } \sigma_{\text {ess }}(S)=\left[\frac{9 b^{2}}{4}, \infty\right) \text { et } \mathcal{N}_{\frac{9 b^{2}}{4}}(S)<\infty,
\end{aligned}
$$

où $\mathcal{N}_{\frac{b^{2}}{4}}(\Delta)$ (resp. $\left.\mathcal{N}_{\frac{9 b^{2}}{4}}(S)\right)$ est le nombre de valeurs propres de $\Delta$ (resp. de $S$ ) inférieures à $\frac{b^{2}}{4}$ (resp. $\grave{a} \frac{9 b^{2}}{4}$ ).

Remarque 0.4. Lorsque $h^{2}+c<0$, plusieurs auteurs ont obtenu des théorèmes d'existence de surfaces de courbure moyenne $h$ dans $\mathbf{M}^{3}(c)$ (cf. [An], [To], [Ne-Sp]): étant donnée une courbe $\gamma$ de classe $C^{\infty}$ plongée dans $\partial_{\infty} \mathbf{M}^{3}(c)$, il existe une surface $M^{2}$ complète (non compacte) et un plongement $i: M^{2} \hookrightarrow \mathbf{M}^{3}(c)$ de courbure moyenne constante $h$ tel que $\gamma$ soit le bord asymptotique de $M$; ce plongement est $C^{\infty}$ jusqu'au bord.

De plus, il est facile de voir que ce plongement est de courbure totale finie. En effet, $\int_{M}\left|\mathcal{A}^{0}\right|^{2} d v$ est invariant par transformation conforme de l'espace ambiant. En se plaçant dans le modèle de la boule et en y considérant la métrique euclidienne, $M$ munie de la nouvelle métrique induite est une surface compacte à bord; la courbure totale est donc finie. On obtient ainsi de nombreux exemples de surfaces satisfaisant aux hypothèses du théorème 0.3 .

Remarque 0.5. Le spectre du Laplacien a été beaucoup étudié sur les surfaces hyperboliques de topologie finie. Dans ce cadre, le résultats portent sur la finitude du nombre de valeurs propres dans un intervalle de la forme $[0, \beta[$, mais il est également possible d'estimer les valeurs propres en fonction de la topologie de la surface et des longueurs des géodésiques périodiques (cf. [D-P-R-S], théorème 1.1 et corollaire 1.3). 
L'organisation de l'article est la suivante. La section 1 est consacrée aux résultats préliminaires et aux notations.

Dans la section 2, on traite d'abord le cas plus simple des surfaces de type anneaux (i.e. homéomorphes à $S^{1} \times \mathbb{R}$ ) en utilisant des théorèmes de comparaison dans le revêtement universel et les propriétés du groupe fondamental.

Dans la section 3, on étudie la topologie et la géométrie de $M$. On montre que $M$ se découpe en une partie compacte et un nombre fini de bouts. Chaque bout étant de type anneau, les résultats de la section 2 permettent d'obtenir des informations géométriques sur $M$.

Dans la section 4 , on démontre le théorème 0.3 en utilisant le découpage précédent.

Remerciements. L'auteur tient à remercier le département de mathématiques de l'Université Fédérale du Ceará, Fortaleza, Brésil, où une partie de ce travail a été effectuée. Ses remerciements vont également à Pierre Bérard et Bruno Colbois pour les discussions autour des problèmes de petites valeurs propres sur les surfaces hyperboliques.

\section{Préliminaires et notations}

On se place dans le cadre des hypothèses du théorème 0.3 , Soit $i: M^{2} \hookrightarrow \mathbf{M}^{3}(c)$ une immersion isométrique où $M$ est un surface complète et orientable, et où $\mathbf{M}^{3}(c)$ est un espace forme de courbure $c<0$ et de dimension 3. Certains des résultats connus cités dans cet article concernent le cas $c=-1$, mais s'étendent facilement au cas $c<0$.

On notera $\mathcal{A}$ la seconde forme fondamentale, $H$ le vecteur courbure moyenne et $\mathcal{A}^{0}=\mathcal{A}-H I d$ la seconde forme fondamentale à trace nulle. La courbure totale est $\int_{M}\left|\mathcal{A}^{0}\right|^{2} d v$.

Soit $N$ un champ de vecteurs unitaire normal à $M$. La courbure moyenne s'écrit $H=h N$ où $h$ est une fonction sur $M$. Dans la suite, on supposera que la fonction $h$ est constante et on choisira $N$ tel que $h \geq 0$. De plus, on supposera que $h^{2}+c<0$ et que la courbure totale est finie; on notera $b=\sqrt{-\left(h^{2}+c\right)}$. Une conséquence importante de la finitude de la courbure totale est le résultat suivant dû à P. Bérard, M. do Carmo et W. Santos (cf. [Be-dC-S2]):

Théorème 1.1. Soit $i: M^{2} \hookrightarrow \mathbf{M}^{3}(c)$ une immersion de courbure moyenne constante telle que $\int_{M}\left|\mathcal{A}^{0}\right|^{2} d v<\infty$. Alors $\lim _{x \rightarrow \infty}\left|\mathcal{A}^{0}\right|(x)=0$.

Une conséquence du théorème 1.1 est que $\left|\mathcal{A}^{0}\right|$ est bornée sur $M$; on a donc $\int_{M}\left|\mathcal{A}^{0}\right|^{\alpha} d v<\infty$ pour tout $\alpha \geq 2$.

L'équation de Gauss sur $M$ s'écrit

$$
\mathcal{K}_{M}=\left(h^{2}+c\right)-\frac{\left|\mathcal{A}^{0}\right|^{2}}{2}=-b^{2}-\frac{\left|\mathcal{A}^{0}\right|^{2}}{2}
$$

où $\mathcal{K}_{M}$ est la courbure sectionnelle de $M$. On a en particulier $\mathcal{K}_{M} \leq-b^{2}$.

Les surfaces de courbure moyenne constante sont points critiques de l'aire pour les déformations qui préservent le volume. L'opérateur de stabilité associé à ce problème variationnel est $S=\Delta-2\left(h^{2}+c\right)-\left|\mathcal{A}^{0}\right|^{2}$. Le fait que les déformations préservent le volume amène à considérer l'opérateur $S$ agissant sur $\mathcal{D}(S)=\{f \in$ $\left.C_{0}^{\infty}(M) \mid \int_{M} f d v=0\right\}$ (cf. [B-dC-E]). Les propriétés spectrales dont il est question dans cet article portent sur des extensions auto-adjointes de $\Delta$ et $S$. L'opérateur 
$\left(\Delta, C_{0}^{\infty}(M)\right)$ est essentiellement auto-adjoint. D'autre part, l'hypothèse de courbure totale finie implique que $(S, \mathcal{D}(S))$ est essentiellement auto-adjoint, et que son unique extension auto-adjointe est égale à celle de $\left(S, C_{0}^{\infty}(M)\right.$ ) (la condition d'intégrale nulle est superflue dans le cadre de l'étude spectrale de $S$; cf. [Ca2], proposition 3.1). Dans la suite, on travaillera donc sur les extensions auto-adjointes de $\left(S, C_{0}^{\infty}(M)\right)$ et $\left(\Delta, C_{0}^{\infty}(M)\right)$, que l'on notera encore $S$ et $\Delta$. Pour un opérateur auto-adjoint $R$, on notera $\sigma(R)$ le spectre de $R, \sigma_{\text {ess }}(R)$ son spectre essentiel, et $\mathcal{N}_{a}(R)$ le nombre de valeurs propres de $R$ qui sont inférieures à $a$.

Les spectres essentiels de $\Delta$ et $S$. Les spectres essentiels de $\Delta$ et de $S$ sont connus (cf. [Ca2], théorème A):

Théorème 1.2. Soit $i: M^{2} \hookrightarrow \mathbf{M}^{3}(c)$ une immersion de courbure moyenne constante telle que $\int_{M}\left|\mathcal{A}^{0}\right|^{2} d v<\infty$. Alors $\sigma_{\mathrm{ess}}(\Delta)=\left[\frac{b^{2}}{4}, \infty\right)$ et $\sigma_{\mathrm{ess}}(S)=\left[\frac{9 b^{2}}{4}, \infty\right)$.

La preuve de ce théorème consiste à montrer d'abord un résultat de compactification (cf. [Ca2], théorème 2.1) qui permette d'étudier le comportement à l'infini de $M$. Les spectres essentiels de $\Delta$ et de $S$ se déduisent alors des propriétés asymptotiques de la métrique.

Le théorème de Lieb. Un outil important de la preuve est le théorème de Lieb. Ce théorème permet de majorer le nombre de valeurs propres négatives d'un opérateur de Schrödinger sur $\mathbb{R}^{n}$ par la norme $L^{\frac{n}{2}}$ du potentiel, lorsque $n \geq 3$ (cf. par exemple [Re-Si], théorème XIII.12). Dans la preuve, la géométrie de $\mathbb{R}^{n}$ intervient uniquement via l'expression du noyau de la chaleur. Il est donc aisé d'obtenir un résultat analogue pour une variété riemannienne satisfaisant une estimée du noyau de la chaleur.

Théorème 1.3. Soit $V$ une variété riemannienne, et soit $R^{c}=\Delta+Q$ un opérateur de Schrödinger sur $V$ avec conditions ${ }^{c}$ sur $\partial V$, où $Q$ est une fonction continue sur $V$. Soit $p_{V}^{c}$ le noyau de la chaleur sur $V$ pour le laplacien avec conditions ${ }^{c}$ sur $\partial V$. Supposons qu'il existe $a \geq 0$ et $\alpha>1$ tels que:

i. $p_{V}^{c}(t, x, x) \leq B t^{-\alpha} \mathrm{e}^{-a t}$;

ii. $Q \in L^{\alpha}(V)$.

Alors

$$
\mathcal{N}_{a}\left(R^{c}\right) \leq C \int_{V}\left|Q_{-}\right|^{\alpha} d v<\infty
$$

ò̀ $Q_{-}(x)=\max \{0,-Q(x)\}$.

Preuve. Soit $\Delta_{a}^{c}=\Delta^{c}-a$ et soit $q_{V}^{c}$ le noyau de la chaleur pour l'opérateur $\Delta_{a}^{c}$. On a en particulier $\mathcal{N}_{a}\left(R^{c}\right)=\mathcal{N}_{0}\left(\Delta_{a}^{c}+Q\right)$. D'autre part, l'hypothèse sur $p_{V}^{c}$ implique que $\Delta_{a}^{c}$ est un opérateur positif dont le noyau $q_{V}^{c}$ vérifie $q_{V}^{c}(t, x, x) \leq B t^{-\alpha}$.

Ces deux propriétés permettent d'imiter la preuve du théorème de Lieb (cf. par exemple [Re-Si], théorème XIII.12) en remplaçant le laplacien par $\Delta_{a}^{c}$. On en déduit que

$$
\mathcal{N}_{a}\left(R^{c}\right)=\mathcal{N}_{0}\left(\Delta_{a}^{c}+Q\right) \leq C \int_{V}\left|Q_{-}\right|^{\alpha} d v<\infty
$$


Remarque 1.4. Dans l'énoncé de ce théorème, l'hypothèse $\alpha>1$ est nécessaire. Par exemple, si $V=\mathbb{R}^{2}$ (on a alors $p_{V}(t, x, x)=(4 \pi t)^{-1}$ ) la conclusion est fausse (cf. [Re-Si], théorème XIII.11). Ceci explique l'hypothèse portant sur la dimension dans l'énoncé original du théorème de Lieb. Dans notre cas, bien qu'on s'intéresse à des variétés de dimension 2 , les estimées obtenues sur les noyaux de la chaleur permettront d'utiliser le théorème 1.3 (cf. propositions 2.4 et 3.2).

\section{LE CAS DES SURFACES DE TYPE ANNEAU}

Avant de traiter le cas des surfaces de courbure moyenne constante, on va montrer deux résultats dans un cadre plus général. Soit $V$ une surface riemannienne; on supposera dans cette section que $V$ satisfait aux hypothèses $\left(\mathrm{H}_{b}\right)$ suivantes:

Hypothèses $\left(\mathrm{H}_{b}\right)$.

- $V$ est homéomorphe à $S^{1} \times \mathbb{R}$ (on dira que $V$ est de type anneau);

- la courbure de $V$ vérifie $\mathcal{K}_{V} \leq-b^{2}$ avec $b>0$;

- chacun des deux bouts de $V$ est de volume infini.

Soit $\Gamma$ le groupe fondamental de $V$, et soit $\pi: \widetilde{V} \rightarrow V$ son revêtement universel. La variété $\widetilde{V}$ munie du rappel de la métrique de $V$ par $\pi$ est une variété de CartanHadamard dont la courbure vérifie $\mathcal{K}_{\widetilde{V}} \leq-b^{2}$. Par un théorème de comparaison "à la Rauch" on obtient l'inégalité suivante:

Lemme 2.1. Pour tout $x \in \widetilde{V}$, notons $\rho_{x}$ la fonction distance sur $\widetilde{V}$ centrée en $x$ : $\rho_{x}(y)=d_{\widetilde{V}}(x, y)$. On $a$

$$
\operatorname{div}\left(\nabla \rho_{x}\right) \geq b \operatorname{coth}\left(b \rho_{x}\right) .
$$

Preuve. Ce résultat est classique, cf. par exemple [Ka].

D'autre part, on déduit des hypothèses $\left(\mathrm{H}_{b}\right)$ qu'il existe sur $V$ une plus petite géodésique périodique $g$; on note $\lambda$ la longueur de $g$. Soit $\tilde{g}$ un relevé de la géodésique $g$ dans $\widetilde{V}$. Le groupe $\Gamma$ est isomorphe à $\mathbf{Z}$, il agit isométriquement sur $\widetilde{V}$ et il est engendré par une isométrie $\tau$ de $\widetilde{V}$ qui est de type hyperbolique et qui fixe $\tilde{g}$ globalement.

Lemme 2.2. Pour tout $x \in \widetilde{V}$ et tout $n \in \mathbb{Z}$, on a

$$
d_{\widetilde{V}}\left(\tau^{n} x, x\right) \geq|n| \lambda
$$

Preuve. Soit $x \in \widetilde{V}$, soit $p_{x}$ sa projection orthogonale sur $\tilde{g}$, et soit $k$ la géodésique de $\widetilde{V}$ orthogonale à $\tilde{g}$ passant par $x$. Pour tout $n \in \mathbb{Z}$, la géodésique $\tau^{n}(k)$ intersecte orthogonalement $\tilde{g}$ en $\tau^{n} p_{x}$. Le minimum de la distance entre $k$ et $\tau^{n}(k)$ est donc $d_{\widetilde{V}}\left(\tau^{n} p_{x}, p_{x}\right)$, et on a

$$
d_{\widetilde{V}}\left(\tau^{n} x, x\right) \geq d_{\widetilde{V}}\left(\tau^{n} p_{x}, p_{x}\right)=|n| \lambda .
$$

Inégalité isopérimétrique et noyau de la chaleur. Les hypothèses $\left(\mathrm{H}_{b}\right)$ permettent d'obtenir l'inégalité isopérimétrique suivante sur $V$ :

Proposition 2.3. Pour tout domaine relativement compact $\Omega \subset V$ on a

$$
b \operatorname{Vol}(\Omega) \leq \operatorname{vol}(\partial \Omega) .
$$


Preuve. Soit $k$ une géodésique de $V$ orthogonale à $g$, soit $\tilde{k}$ un relevé de $k$ dans $\tilde{V}$, et soit $D$ le domaine de Dirichlet de l'action de $\Gamma$ délimité par $\tilde{k}$ et $\tau(\tilde{k})$.

Soit $\Omega$ un domaine relativement compact de $V$ et soit $\widetilde{\Omega}$ son relevé dans $D$. Le bord de $\widetilde{\Omega}$ se décompose en

$$
\partial \widetilde{\Omega}=\widetilde{\partial \Omega} \cup B_{\Omega} \cup \tau\left(B_{\Omega}\right)
$$

où $\widetilde{\partial \Omega}$ est le relevé de $\partial \Omega$ dans $D$ et $B_{\Omega}=\tilde{k} \cap \widetilde{\Omega}$.

Soit $n \in \mathbb{N}$, et soit

$$
\widetilde{\Omega}_{n}=\bigcup_{j=0}^{n} \tau^{j}(\widetilde{\Omega})
$$

On a

$$
\partial \widetilde{\Omega}_{n}=\bigcup_{j=0}^{n} \tau^{j}(\widetilde{\partial \Omega}) \cup B_{\Omega} \cup \tau^{n+1}\left(B_{\Omega}\right) .
$$

En intégrant l'inégalité (2.1) sur un domaine $E \subset \widetilde{V}$ et en utilisant la formule de Stokes on obtient $b \operatorname{Vol}(E) \leq \operatorname{vol}(\partial E)$ pour tout domaine $E$ relativement compact dans $\widetilde{V}$. On a donc

$$
b \operatorname{Vol}\left(\widetilde{\Omega}_{n}\right) \leq \operatorname{vol}\left(\partial \widetilde{\Omega}_{n}\right)
$$

d'où

$$
(n+1) b \operatorname{Vol}(\Omega) \leq(n+1) \operatorname{vol}(\partial \Omega)+2 \operatorname{vol}\left(B_{\Omega}\right) .
$$

Le résultat s'obtient en divisant par $n+1$ et en faisant tendre $n$ vers l'infini.

Notons $p_{V}$ et $p_{\widetilde{V}}$ les noyaux minimaux de l'équation de la chaleur pour le laplacien sur $V$ et $\widetilde{V}$. On a sur $p_{V}$ l'estimée suivante:

Proposition 2.4. Pour tout $x \in V$ et tout $t>0$, on a

$$
p_{V}(t, x, x) \leq A t^{-\frac{3}{2}} \mathrm{e}^{-\frac{b^{2} t}{4}}
$$

où $A$ est une constante indépendante de $x$.

Preuve. Soit $q_{b}$ le noyau de la chaleur sur $\mathbb{H}^{2}\left(-b^{2}\right)$, le plan hyperbolique de courbure $-b^{2}$. Pour des raisons de symétrie, on peut écrire

$$
q_{b}(t, x, y)=G_{b}\left(t, \cosh \left(b r_{x}(y)\right)\right)
$$

où $G_{b}(t, s)$ est une fonction sur $\mathbb{R}_{+}^{2}$, et $r_{x}$ est la fonction distance sur $\mathbb{H}^{2}\left(-b^{2}\right)$ centrée en $x$. Comme $q_{b}$ est solution de l'équation de la chaleur sur $\mathbb{H}^{2}\left(-b^{2}\right)$, on a

$$
\frac{\partial G_{b}}{\partial t}=2 b^{2} s \frac{\partial G_{b}}{\partial s}+b^{2}\left(s^{2}-1\right) \frac{\partial^{2} G_{b}}{\partial s^{2}}
$$

De plus, on a $\frac{\partial G_{b}}{\partial s} \leq 0$ et $\frac{\partial^{2} G_{b}}{\partial s^{2}} \geq 0$ (cela se déduit par exemple de l'expression de $q_{b}$; cf. [Da]). Soit $I_{b}$ la fonction sur $\mathbb{R}_{+} \times \widetilde{V} \times \widetilde{V}$ définie par

$$
I_{b}(t, x, y)=G_{b}\left(t, \cosh \left(b \rho_{x}(y)\right)\right)
$$

où $\rho_{x}$ est la fonction distance sur $\widetilde{V}$ centrée en $x$. On a

$$
\Delta_{y} I_{b}+\frac{\partial I_{b}}{\partial t}=-\operatorname{div}\left(\nabla \cosh \left(b \rho_{x}\right)\right) \frac{\partial G_{b}}{\partial s}-b^{2}\left(\cosh ^{2}\left(b \rho_{x}\right)-1\right) \frac{\partial^{2} G_{b}}{\partial s^{2}}+\frac{\partial G_{b}}{\partial t} .
$$


De l'inégalité (2.1) et des propriétés de la fonction $G_{b}$ on déduit que $\Delta_{y} I_{b}+\frac{\partial I_{b}}{\partial t} \geq 0$. Par un résultat classique (cf. par exemple [C-L-Y], proposition 1) on a alors $p_{\tilde{V}} \leq I_{b}$, et par les majorations connues du noyau de la chaleur dans $\mathbb{H}^{2}\left(-b^{2}\right)$ (cf. [Da-Ma], théorème 3.1 ), on obtient pour $p_{\tilde{V}}$ la majoration suivante:

$$
p_{\widetilde{V}}(t, \tilde{x}, \tilde{y}) \leq C t^{-\frac{3}{2}} \mathrm{e}^{-\frac{b^{2} t}{4}}\left(1+b d_{\widetilde{V}}(\tilde{x}, \tilde{y})\right) \mathrm{e}^{-\frac{b}{2} d_{\widetilde{V}}(\tilde{x}, \tilde{y})}
$$

D'autre part, le noyau $p_{V}$ se déduit de $p_{\widetilde{V}}$ (cf. [Bo], proposition 2.6):

$$
p_{V}(t, x, y)=\sum_{\gamma \in \Gamma} p_{\widetilde{V}}(t, \gamma \tilde{x}, \tilde{y})=\sum_{n \in \mathbb{Z}} p_{\widetilde{V}}\left(t, \tau^{n} \tilde{x}, \tilde{y}\right)
$$

où $\tilde{x}$ et $\tilde{y}$ sont des relevés de $x$ et $y$ dans $\widetilde{V}$. La majoration (2.3) donne alors

$$
p_{V}(t, x, y) \leq C t^{-\frac{3}{2}} \mathrm{e}^{-\frac{b^{2} t}{4}} \sum_{n \in \mathbb{Z}}\left(1+b d_{\tilde{V}}\left(\tau^{n} \tilde{x}, \tilde{y}\right)\right) \mathrm{e}^{-\frac{b}{2} d_{\widetilde{V}}\left(\tau^{n} \tilde{x}, \tilde{y}\right)}
$$

Notons $f(u)=(1+b u) \mathrm{e}^{-\frac{b}{2} u} ;$ soit $A_{0}=\sup \left\{f(u) \mid u \in \mathbb{R}_{+}\right\}$, et soit $n_{0} \in \mathbb{N}$ tel que $f$ soit décroissante sur $\left[n_{0} \lambda, \infty[\right.$. Par le lemme 2.2 on obtient

$$
\sum_{n \in \mathbb{Z}}\left(1+b d_{\widetilde{V}}\left(\tau^{n} \tilde{x}, \tilde{x}\right)\right) \mathrm{e}^{-\frac{b}{2} d_{\widetilde{V}}\left(\tau^{n} \tilde{x}, \tilde{x}\right)} \leq\left(2 n_{0}+1\right) A_{0}+\sum_{|n|>n_{0}}(1+b|n| \lambda) \mathrm{e}^{-\frac{b}{2}|n| \lambda}
$$

d'où

$$
p_{V}(t, x, x) \leq A t^{-\frac{3}{2}} \mathrm{e}^{-\frac{b^{2} t}{4}}
$$

où la constante $A$ est indépendante de $x$.

Remarque 2.5. La constante $A$ donnée par la proposition 2.4 pour la majoration de $p_{V}(t, x, x)$ n'est pas universelle; elle dépend de la géométrie de $M$ à travers $\lambda$. En particulier, $A$ tend vers l'infini lorsque $\lambda$ tend vers 0 .

Spectres de $S$ et du laplacien. Soit $i: M^{2} \hookrightarrow \mathbf{M}^{3}(c)$ une immersion de courbure moyenne constante $h$ telle que $h^{2}+c<0$ et $\int_{M}\left|\mathcal{A}^{0}\right|^{2} d v<\infty$. On suppose dans cette section que $M$ est de type anneau et on montre un résultat annalogue aux théorèmes 4.1 et $\mathrm{B}$ de $\mathrm{Ca} 2$.

L'équation de Gauss (1.1) implique en particulier que $M$ est de courbure négative majorée par $-b^{2}$. De plus, chacun de deux bouts de $M$ est de volume infini (c'est par exemple une conséquence de [Ca2], proposition 2.5).

La surface $M$ satisfait donc aux hypothèses $\left(\mathrm{H}_{b}\right)$, et on peut utiliser les résultats précédents. De la propositions 2.3 et de l'inégalité de Cheeger on déduit que $\sigma(\Delta) \subset$ $\left[\frac{b^{2}}{4}, \infty\right)$, et la proposition [2.4 permet d'appliquer le théorème [1.3] à l'opérateur $T=$ $\Delta-\left|\mathcal{A}^{0}\right|^{2}$. En tenant compte du théorème 1.2 on obtient le résultat suivant:

Théorème 2.6. Soit $i: M^{2} \hookrightarrow \mathbf{M}^{3}(c)$ une immersion de courbure moyenne constante telle que $h^{2}+c<0$ avec $M$ de type anneau; on note $b=\sqrt{-\left(h^{2}+c\right)}$. Si $\int_{M}\left|\mathcal{A}^{0}\right|^{2} d v<\infty$ alors:

i. $\sigma(\Delta)=\left[\frac{b^{2}}{4}, \infty\right)$;

ii. $\sigma_{\mathrm{ess}}(S)=\left[\frac{9 b^{2}}{4}, \infty\right)$ et il existe une constante $c_{M}$ telle que

$$
\mathcal{N}_{\frac{9 b^{2}}{4}}(S) \leq c_{M} \int_{M}\left|\mathcal{A}^{0}\right|^{3} d v<\infty .
$$


Remarque 2.7. La constante $c_{M}$ dépend de la géométrie de $M$ à travers l'estimée du noyau de la chaleur obtenue à la proposition 2.4. Compte tenu de la remarque 2.5. $c_{M}$ dépend de la longueur $\lambda$ de la plus petite géodésique périodique de $M$.

Le théorème 2.6] s'applique en particulier aux surfaces de révolution de courbure moyenne constante $h<1$ dans $\mathbb{H}^{3}$ (les surfaces de Delaunay). Pour $h$ fixé, on a une famille à un paramètre de telle surfaces. Dans cette famille, la valeur de $\lambda$ peut être rendue arbitrairement petite, et la valeur de la constante $c_{M}$ peut donc être rendue arbitrairement grande.

\section{GÉométrie Des bouts}

Soit $i: M^{2} \hookrightarrow \mathbf{M}^{3}(c)$ une immersion de courbure moyenne constante $h$ telle que $h^{2}+c<0$ et $\int_{M}\left|\mathcal{A}^{0}\right|^{2} d v<\infty$. Dans cette section, on étudie la topologie de $M$ et on utilise les résultats de la section 2 pour étudier la géométrie dans chaque bout de $M$.

Type topologique de $M$. La finitude de la courbure totale a des conséquences topologiques sur $M$ : il existe un compact $K \subset M$ et un difféomorphisme

$$
\Psi:\left\{\begin{aligned}
\partial K \times] 0, \infty[ & \rightarrow M \backslash K \\
(y, t) & \mapsto \Psi(y, t)
\end{aligned}\right.
$$

Cf. par exemple Ca2, théorème 2.1, pour la construction de $K$ et $\Psi$. On en déduit en particulier que $M$ est homéomorphe à une surface compacte privée d'un nombre fini de disques.

De plus, dans les coordonnées $(y, t)$, la métrique de $M$ s'écrit

$$
g_{M}=\mu^{2} d y^{2}+\nu^{2} d t^{2}
$$

où $\mu$ et $\nu$ sont des fonctions sur $M$ qui vérifient $\lim _{\infty} \frac{1}{\mu} \frac{\partial \mu}{\partial t}=1$ et $\lim _{\infty} \nu^{2}=-\left(h^{2}+c\right)$ (cf. [Ca2], proposition 2.5). On en déduit en particulier que chaque bout de $M$ est de volume infini.

D'autre part, l'équation de Gauss (1.1) implique que $M$ est de courbure négative. Il existe donc dans chaque bout une plus petite géodésique périodique, et en découpant $M$ le long de ces géodésiques on a

$$
M=D \cup E_{1} \cup \ldots \cup E_{n}
$$

où $D$ est compact, chaque $E_{i}$ est homéomorphe à $S^{1} \times \mathbb{R}_{+}$, et les bords de $D$ et des $E_{i}$ sont des géodésiques fermées de $M$.

Inégalité isopérimétrique et noyau de la chaleur dans les bouts. Soit $E$ un bout de $M$ et soit $\gamma=\partial E$. Considérons sur $E$ les coordonnées de Fermi relatives à la géodésique $\gamma$ : les coordonnées de $x \in E$ sont l'abscisse $s(x)$ de la projection de $x$ sur $\gamma$, et la distance $r(x)$ entre $x$ et $\gamma$.

On peut alors écrire $E=\left(S^{1} \times \mathbb{R}_{+}, g_{E}\right)$, où $g_{E}$ est la métrique de $M$ restreinte à $E$ exprimée dans les coordonnées $(s, r)$. Soit $V=S^{1} \times \mathbb{R}$, et soit $\sigma: V \rightarrow V$ définie par $\sigma(s, r)=(s,-r)$. On note $g_{V}$ la métrique définie par

$$
g_{V}=\left\{\begin{aligned}
g_{E} & \text { sur } S^{1} \times \mathbb{R}_{+}, \\
\sigma^{*} g_{E} & \text { sur } S^{1} \times \mathbb{R}_{-} .
\end{aligned}\right.
$$

La métrique $g_{V}$ ainsi définie est de classe $C^{2}$, et la surface $V$ munie de cette métrique satisfait aux hypothèses $\left(\mathrm{H}_{b}\right)$. 
En appliquant les résultats de la section précédente à la surface $V$, on obtient les deux propositions suivantes:

Proposition 3.1. Soit $E$ un bout de $M$. Pour tout domaine relativement compact $\Omega \subset E$ on $a$

$$
b \operatorname{Vol}(\Omega) \leq \operatorname{vol}(\partial \Omega)
$$

Preuve. La preuve est une conséquence de la proposition 2.3 car $\Omega \subset V$.

Proposition 3.2. Soit $E$ un bout de $M$ et soit $p_{E}^{N}$ le noyau de la chaleur sur $E$ avec condition de Neumann sur $\partial E$. On a

$$
p_{E}^{N}(t, x, x) \leq B t^{-\frac{3}{2}} \mathrm{e}^{-\frac{b^{2} t}{4}}
$$

où $B$ est une constante indépendante de $x$.

Preuve. Soit $F$ la fonction définie sur $\mathbb{R}_{+} \times E \times E$ par

$$
F(t, x, y)=p_{V}(t, x, y)+p_{V}(t, \sigma x, y) .
$$

Comme $p_{V}$ satisfait à l'équation de la chaleur dans $V$, on a aussi

$$
\Delta_{y} F+\frac{\partial F}{\partial t}=0
$$

sur $E$. Soit $f$ une fonction définie sur $E$, en utilisant l'invariance de $p_{V} \operatorname{par} \sigma$ on obtient

$$
\begin{aligned}
\int_{E} F(t, x, y) f(y) d y & =\int_{E} p_{V}(t, x, y) f(y) d y+\int_{E} p_{V}(t, \sigma x, y) f(y) d y \\
& =\int_{E} p_{V}(t, x, y) f(y) d y+\int_{\sigma(E)} p_{V}(t, \sigma x, \sigma y) f(\sigma y) d y \\
& =\int_{V} p_{V}(t, x, y) \bar{f}(y) d y .
\end{aligned}
$$

où $\bar{f}(y)=f(y)$ si $y \in E$ et $\bar{f}(y)=f(\sigma y)$ si $y \in \sigma(E)$. Soit $x \in E$, lorsque $t$ tend vers 0 , on obtient

$$
\lim _{t \rightarrow 0} \int_{E} F(t, x, y) f(y) d y=\bar{f}(x)=f(x) .
$$

D'autre part, en utilisant l'invariance de $p_{V}$ par $\sigma$, on obtient

$$
\begin{aligned}
\nabla_{y} F(t, x, y) & =\nabla_{y} p_{V}(t, x, y)+\nabla_{y} p_{V}(t, \sigma x, y) \\
& =\nabla_{y} p_{V}(t, x, y)+\sigma_{*}\left(\nabla_{y} p_{V}(t, x, \sigma y)\right) .
\end{aligned}
$$

En tout point $y \in \partial E, \sigma_{*}$ est la réflexion par rapport à l'espace tangent à $\partial E$; on en déduit que la dérivée normale de $F$ est nulle le long de $\partial E$. De cette condition au bord, et des équations (3.2) et (3.3) on déduit

$$
p_{E}^{N}(t, x, y)=F(t, x, y)=p_{V}(t, x, y)+p_{V}(t, \sigma x, y) .
$$

Par la proposition 2.4 on a $p_{V}(t, x, x) \leq A t^{-\frac{3}{2}} \mathrm{e}^{-\frac{b^{2} t}{4}}$. Soient $\tilde{x}$ et $\widetilde{\sigma x}$ des relevés de $x$ et $\sigma x$ appartenant à un même domaine fondamental de $\widetilde{V}$. Les points $\tilde{x}$ et $\widetilde{\sigma x}$ appartiennent à une même géodésique orthogonale à $\tilde{g}$. Par une preuve similaire à celle du lemme 2.2, on a $d_{\widetilde{V}}\left(\tau^{n} \tilde{x}, \widetilde{\sigma x}\right) \geq|n| \lambda$ et la majoration (2.5) donne $p_{V}(t, x, \sigma x) \leq A t^{-\frac{3}{2}} \mathrm{e}^{-\frac{b^{2} t}{4}}$. Finalement, on obtient $p_{E}^{N}(t, x, x) \leq 2 A t^{-\frac{3}{2}} \mathrm{e}^{-\frac{b^{2} t}{4}}$. 


\section{Preuve Du théorème 0.3}

Soit $i: M^{2} \hookrightarrow \mathbf{M}^{3}(c)$ une immersion de courbure moyenne constante $h$ telle que $h^{2}+c<0$ et $\int_{M}\left|\mathcal{A}^{0}\right|^{2} d v<\infty$. La preuve du théorème 0.3 repose sur la méthode d'encadrement Dirichlet-Neumann:

Proposition 4.1. Soit $V$ une variété riemannienne, et soit $R=\Delta+Q$ un opérateur de Schrödinger sur $V$, où $Q$ est continue. Supposons qu'on ait un découpage de $V$ :

$$
V=\bar{\Omega}_{1} \cup \bar{\Omega}_{2} \cup \ldots \cup \bar{\Omega}_{n}
$$

où les $\Omega_{i}$ sont des ouverts disjoints. Notons $R_{\Omega_{i}}^{D}$ (resp. $R_{\Omega_{i}}^{N}$ ) l'opérateur $R$ agissant sur $\Omega_{i}$ avec condition de Dirichlet (resp. de Neumann) sur $\partial \Omega_{i}$. On a

$$
\sum_{i=1}^{n} \mathcal{N}_{a}\left(R_{\Omega_{i}}^{D}\right) \leq \mathcal{N}_{a}(R) \leq \sum_{i=1}^{n} \mathcal{N}_{a}\left(R_{\Omega_{i}}^{N}\right)
$$

Preuve. Ce résultat est classique, cf. par exemple [Re-Si], section XIII.15, ou [Be], section III.C.

Preuve du théorème $\mathbf{0 . 3}$. Les spectres essentiels de $\Delta$ et $S$ sont connus (cf. théorème [1.2). Pour le point $i$, on utilise le découpage (3.1). Pour un domaine $\Omega \subset M$, notons $\Delta_{\Omega}^{N}$ le laplacien sur $\Omega$ avec condition de Neumann au bord. La méthode d'encadrement Dirichlet-Neumann donne

$$
\mathcal{N}_{\frac{b^{2}}{4}}(\Delta) \leq \mathcal{N}_{\frac{b^{2}}{4}}\left(\Delta_{D}^{N}\right)+\mathcal{N}_{\frac{b^{2}}{4}}\left(\Delta_{E_{1}}^{N}\right)+\ldots+\mathcal{N}_{\frac{b^{2}}{4}}\left(\Delta_{E_{n}}^{N}\right)
$$

Comme $D$ est compact, $\mathcal{N}_{\frac{b^{2}}{4}}\left(\Delta_{D}^{N}\right)$ est fini; pour chaque $E_{i}, i=1, \ldots, n$, la proposition 3.1 et l'inégalite de Cheeger montrent que $\mathcal{N}_{\frac{b^{2}}{4}}\left(\Delta_{E_{i}}^{N}\right)=0$. Le point i est donc prouvé.

Pour le point ii, on a par la méthode d'encadrement Dirichlet-Neumann

$$
\mathcal{N}_{\frac{9 b^{2}}{4}}(S) \leq \mathcal{N}_{\frac{9 b^{2}}{4}}\left(S_{D}^{N}\right)+\mathcal{N}_{\frac{9 b^{2}}{4}}\left(S_{E_{1}}^{N}\right)+\ldots+\mathcal{N}_{\frac{9 b^{2}}{4}}\left(S_{E_{n}}^{N}\right)
$$

Comme $D$ est compact, $\mathcal{N}_{\frac{9 b^{2}}{4}}\left(S_{D}^{N}\right)$ est fini. Sur chaque $E_{i}, i=1, \ldots, n$, la proposition 3.2 permet d'appliquer le théorème 1.3 à l'opérateur $T=\Delta-\left|\mathcal{A}^{0}\right|^{2}$. On en déduit que

$$
\mathcal{N}_{\frac{9 b^{2}}{4}}\left(S_{E_{i}}^{N}\right)=\mathcal{N}_{\frac{b^{2}}{4}}\left(T_{E_{i}}^{N}\right) \leq C \int_{E_{i}}\left|\mathcal{A}^{0}\right|^{3} d v<\infty .
$$

Ceci termine la preuve du théorème 0.3

\section{RÉFÉRENCES}

[An] Anderson, M.- Complete minimal varieties in hyperbolic space. Invent. Math. 69 (1982), 477-494. MR 84c:53005

[B-dC-E] Barbosa, J.L.; do Carmo, M.; Eschenburg, J.- Stability of hypersurfaces of constant mean curvature in Riemannian manifolds. Math. Z. 197 (1988), 123-138. MR 88m:53109

[Be] BÉRARD, P.- Spectral geometry: direct and inverse problems, Lecture Note in Math. 1207, Springer Verlag 1986. MR 88f:58146

[Be-dC-S1] Bérard, P.; do Carmo, M.; Santos, W.- The index of constant mean curvature surfaces in hyperbolic 3-space. Math. Z. 224 (1997), 313-326. MR 98a:53008

[Be-dC-S2] Bérard, P.; Do CARmo, M.; SAntos, W.- Complete hypersurfaces with constant mean curvature and finite total curvature. Ann. Global Anal. Geom. 16 (1998), 273290. MR 2000d:53093 
[Bo] Bordoni, M.- Comparing heat operators through local isometries or fibrations, Prépublication de l'E.N.S. Lyon $n^{\circ}$ 190, 1996.

[dC-dS] Do Carmo, M.; da Silveira, A.M.- Index and total curvature of surfaces with constant mean curvature. Proc. Amer. Math. Soc. 110 (1990), 1009-1015. MR 91c:53055

[Ca1] Castillon, P.- Sur l'opérateur de stabilité des sous-variétés à courbure moyenne constante dans l'espace hyperbolique. Manuscripta Math., 94 (1997) 385-400. MR 99c:53062

[Ca2] Castillon, P.- Spectral properties of constant mean curvature submanifolds in hyperbolic space. Ann. Global Anal. Geom., 17 (1999) 563-580. MR 2000j:53079

[C-L-Y] Cheng, S.S.; Li, P.; YAu, S.T.- Heat equations on minimal submanifolds and their applications. Am. J. Math. 106 (1984), 1033-1065. MR 85m:58171

[Da] Davies, E.B.- Heat kernels and spectral theory, Cambridge University Press, 1989. MR 90e:35123

[Da-Ma] Davies, E.B.; Mandouvalos, N.- Heat kernel bounds on hyperbolic space and Kleinian groups. Proc. London Math. Soc. 57 (1988), 182-208. MR 89i:58137

[D-P-R-S] Dodziuk, J.; Pignataro, T.; Randol, B.; Sullivan, D.- Estimating small eigenvalues of Riemann surfaces. In: The legacy of Sonya Kovalevskaya, 93-121, Contemp. Math. 64, AMS, Providence, RI, 1996. MR 88h:58119

[FC] Fischer-Colbrie, D.- On complete minimal surfaces with finite Morse index in threemanifold. Invent. Math. 82 (1985), 121-132. MR 87b:53090

[Ka] KARChER, H.- Riemannian comparison constructions. In: Global Differential Geometry vol. 27, 170-222, MAA studies in Math. 2nd ed., 1989. MR 91b:53046

[Ne-Sp] Nelli, B.; Spruck, J.- On existence and uniqueness of constant mean curvature hypersurfaces in hyperbolic space. In: Geometric analysis and the calculus of variations, 253-266, Internat. Press, Cambridge, MA, 1996. MR 98e:53106

[Re-Si] Reed, M.; Simon, B.- Methods of modern mathematical physics, Academic Press, 1978, 1979, 1980, 1975 (vol. I to IV) MR 58:12429a MR 58:12429b MR 80m:81085 MR 58:12429c

[dS] A.M. DA Silveira - Stability of complete noncompact surfaces with constant mean curvature. Math. Ann. 277 (1987), 629-638. MR 88h:53053

[To] TONEGAWA, Y.- On existence and regularity of constant mean curvature hypersurfaces in hyperbolic space. Math. Z. 221 (1996), 591-615. MR 97c:53016

Institut Fourier, B.P. 74, 38402 Saint Martin D'Hères Cedex, France

E-mail address: philippe.castillon@ujf-grenoble.fr

Current address: Département des Sciences Mathématiques, cc 51, Université Montpellier 2, 34095 Montpellier cedex 5, France

E-mail address: philippe.castillon@math.univ-montp2.fr 